\title{
Optimizing GNSS Navigation Data Message Decoding in Urban Environment
}

\author{
Marion Roudier, Thomas Grelier, Lionel Ries \\ Radionavigation Signals and Equipments \\ CNES \\ Toulouse, France \\ roudier@recherche.enac.fr, Thomas.Grelier@cnes.fr, \\ Lionel.Ries@cnes.fr
}

\author{
Axel Garcia-Pena, Olivier Julien \\ SIGNAV Research Group \\ ENAC \\ Toulouse, France
}

garcia-pena@recherche.enac.fr, ojulien@recherche.enac.fr

\author{
Charly Poulliat, Marie-Laure Boucheret \\ INPT-ENSEEIHT/IRIT \\ University of Toulouse \\ Toulouse, France \\ charly.poulliat@enseeiht.fr, Marie- \\ Laure.Boucheret@enseeiht.fr
}

\author{
Damien Kubrak \\ Thales Alenia Space \\ Toulouse, France \\ damien.kubrak@thalesaleniaspace.com
}

\begin{abstract}
Nowadays, the majority of new GNSS applications targets dynamic users in urban environments; therefore the decoder input in GNSS receivers needs to be adapted to the urban propagation channel to avoid mismatched decoding when using soft input channel decoding. The aim of this paper consists thus in showing that the GNSS signals demodulation performance is significantly improved integrating an advanced soft detection function as decoder input in urban areas. This advanced detection function takes into account takes into account some a priori information on the available Channel State Information (CSI). If no CSI is available, one has to blindly adapt the detection function in order to operate close to the perfect CSI case. This will lead to avoid mismatched decoding due to, for example, the consideration by default of the Additive White Gaussian Noise (AWGN) channel for the derivation of soft inputs to be fed to soft input decoders. As a consequence the decoding performance will be improved in urban areas. The expressions of the soft decoder input function adapted for an urban environment is highly dependent on the available CSI at the receiver end. Based on different model of urban propagation channels, several CSI contexts will be considered namely perfect CSI, partial statistical CSI and no CSI. Simulation results will be given related to the GPS L1C demodulation performance with these different advanced detection function expressions in an urban environment. The results presented in this paper are valid for any kind of soft input decoders, such as Viterbi decoding for trellis based codes, the MAP/BCJR decoding for turbo-codes and the Belief Propagation decoding for LDPC codes.
\end{abstract}

Keywords-GNSS; channel decoding; soft input decoders; Channel State Information; urban propagation channel model; GNSS demodulation performance

\section{INTRODUCTION}

Global Navigation Satellite Systems (GNSS) are increasingly present in our everyday life. The interest of new users with further operational needs implies a constant evolution of the current GNSS systems. A significant part of the new applications are found in environments with difficult reception conditions such as urban or indoor areas. In these obstructed environments, the received signal is severely impacted by obstacles which induce fading of the resulting received signal that is detrimental to both the ranging and demodulation capability of the receiver. In order to protect the useful information against these errors potentially brought by the urban environment, a channel code is applied.

To be able to ensure the principal GNSS function consisting in providing positioning capabilities, the receiver needs to access the useful information transmitted by the GNSS signal, called the navigation message. The navigation message is structured in different basic units of information, called codewords. Each codeword, in addition to containing useful information, carries redundant bits which are the result of applying a channel code to the useful information bits. The reason of introducing a channel code is to protect these information bits against errors introduced by the propagation channel. Consequently, at the reception, the GNSS navigation message, or more especially the associated codewords, needs to be decoded by the receiver to reliably recover the transmitted useful bits.

Therefore, channel coding introduction is essential to allow a better rate of data demodulation success in difficult environments. This process is very sensitive to the correct computation of the detection function which will feed the decoder input. Significant improvements are obtained by considering soft detection enabling the use of soft input channel decoders. In this context, the channel decoders need to be fed by soft inputs that are sufficient statistics from the detection point of view. This is usually achieved by computing Log-Likelihood Ratios (LLRs) based on both observation samples and channel parameters. Usually, without any a priori information on the encountered channel, the expression of the detection function in GNSS receivers is obtained assuming an Additive White Gaussian Noise (AWGN) propagation channel. However since we are interested by urban applications, the 
detection function expression should be adapted to the urban propagation channel in order to improve the GNSS signals decoding performance and to avoid mismatched decoding errors (providing by the use of a detection function not computed for the considered propagation channel).

The aim of this paper is thus to propose an advanced processing algorithm in order to improve the receiver sensibility in urban channels. More specifically we have tried to determine whether the decoder used in classical GNSS receivers with a detection function computed for an AWGN channel model is satisfactory in urban environments, or whether it is worthwhile to integrate an advanced detection function adapted to an urban channel model, in order to improve the GNSS signals decoding performance in urban environments.

The soft detection function at the GNSS decoder input has been derived according to different levels about the propagation channel fading behavior knowledge called Channel State Information (CSI), in urban environments. Three configurations have been taken into account in this paper for the detection function derivation based on analytical or approximated LLR derivations:

- $\quad$ Perfect CSI,

- $\quad$ Statistical CSI

- $\quad$ No CSI.

In order to numerically determine the GNSS signal decoding gain obtained by the adaptation of the detection function to an urban environment, the detection function expressions derived from the three use cases listed above have been integrated in the Simulator for GNSS Message Performance SiGMeP described in [1]. The results obtained with the refined detection function expressions will be compared with those obtained with a classical decoder using the detection function corresponding to the AWGN channel model.

The paper is organized as follows. Section II introduces the soft input channel decoding concept and describes the received symbol modeling of GNSS signals in urban environments. Section III details the derivation of the soft detection function expressions for perfect CSI, statistical partial CSI and no CSI. Section IV presents the simulation conditions and the results obtained with SiGMeP. Finally, conclusions are given in Section V.

\section{SYSTEM MODEL}

\section{A. Soft Input Channel Decoding}

The navigation message, protected against errors by channel coding, is carried by the propagation channel. At the receiver end, the decoder uses a decoding algorithm in order to recover the transmitted useful bits. The channel decoding algorithms considered in this paper are based on soft inputs generally using Log-Likelihood Ratios (LLRs) based on A Posteriori Probability (APP)[2][3]. Indeed, most of existing soft input channel decoding algorithms such as Viterbi decoding of trellis based codes, or message passing algorithms based on sub-optimal decoding algorithms like the Belief
Propagation for the LDPC codes, or BCJR for turbo-codes, are using LLRs or related approximated expressions as soft inputs.

For binary random variables as inputs (which is the case in GNSS), the LLR [2] is defined as follows:

$$
L L R=\log \left(\frac{p(x=+1 / y)}{p(x=-1 / y)}\right)
$$

Where:

- $\quad p(x / y)$ is the a posteriori probability that the symbol $x$ has been transmitted, knowing the received symbol $y$.

This LLR is thus the detection function at the decoder input which will be derived in the urban environment case for this work. Using the Bayes rule and since we assume that $p(x=$ $+1)=p(x=-1)=\frac{1}{2}$ in our context, the LLR expression can finally rewritten as:

$$
L L R=\log \left(\frac{p(y / x=+1)}{p(y / x=-1)}\right)
$$

Where $p(y / x)$ is the transition probability density function associated with the channel.

This is the soft detection function expression that will be derived in Section III according to the CSI knowledge level.

\section{B. Received symbol modeling}

In a GNSS receiver, the received symbol $y$ to be decoded corresponds to the correlator output of the $I$ channel of the data component $I P$ modeled by this expression:

$$
I P[k]=\frac{a[k]}{2} x[k] \cos \left(\varepsilon_{\theta}[k]\right)+n_{I}[k]
$$

Where:

- $\quad k$ is the symbol index,

- $a$ is the received signal amplitude at the receiver antenna output,

- $\quad x$ is the emitted symbol,

- $\varepsilon_{\theta}$ is the carrier phase tracking error,

- $n_{I}$ is the additive Gaussian noise at the correlator output. $n_{I}$ follows thus a centered Gaussian distribution: $N\left(0, \sigma_{n_{I}}^{2}\right)$, with $\sigma_{n_{I}}^{2}$ equal to:

$$
\sigma_{n_{I}}^{2}=\frac{N_{0}}{4 T I}
$$

Where: 
- $N_{0}$ is the noise spectral density,

- $T I$ is the integration duration.

Since the correlator output $I P$ is normalized before feeding the decoder, the decoder input model can be written as:

$$
y[k]=I P[k]=a[k] x[k] \cos \left(\varepsilon_{\theta}[k]\right)+n_{I}{ }^{\prime}[k]
$$

With:

$$
\sigma_{n_{I}^{\prime}}^{2}=\frac{N_{0}}{T I}
$$

In our context, we assume perfect interleaving before transmission. As a result, the fading process associated with $a[k]$ will be considered as an uncorrelated fading process.

\section{DERIVATION OF THE SOFT DETECTION FunCTION}

By default, if no a priori knowledge on the channel is available at the receiver end, the detection function which is implemented in GNSS receivers is generally computed assuming an AWGN propagation channel as the default channel. In this work urban environments are targeted thus it seems more adapted to compute the detection function considering an urban propagation channel. This derivation highly depends on the CSI available at the receiver and on the channel parameters which can be estimated.

The detection function will be firstly reviewed for the AWGN propagation channel case, as it is used in the current GNSS receivers. Then, according to the knowledge of the propagation channel fading parameters, the detection function will be derived for mainly three different cases. The first case assumes a perfect CSI knowledge, the second considers a partial knowledge of statistical CSI, and the third case corresponds to no a priori CSI.

\section{A. LLR Expression in the AWGN Case}

The detection function used classically in the GNSS context corresponds to the AWGN propagation channel model. In this channel model, the received symbol is written as:

$$
y[k]=x[k]+n_{I}^{\prime}[k]
$$

From (7) we can write:

$$
p(y[k] / x[k])=\frac{1}{\sqrt{2 \pi \sigma^{2} n_{I}^{\prime}}} e^{\frac{-(y[k]-x[k])^{2}}{2 \sigma^{2} n_{I}}}
$$

And thus from (2) we can deduce that:

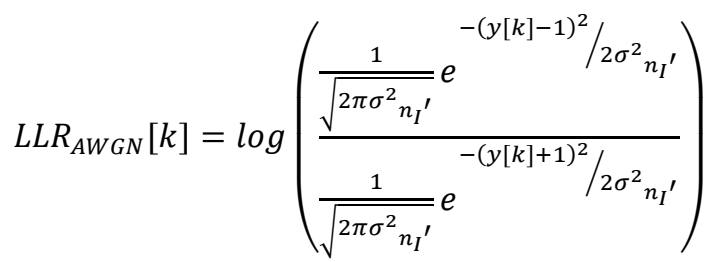

And thus, as classically known, we have:

$$
L L R_{A W G N}[k]=\frac{2 y[k]}{\sigma^{2}{ }_{n_{I}}{ }^{\prime}}
$$

Note that it is implicitly assumed that the signal to noise ratio can be estimated, since the corresponding $L L R$ is a scaled version of the observation by a constant proportional to the signal to noise ratio. Considering this model at the receiver is equivalent to consider that we only have access to the average signal-to-noise ratio for the received signal model of equation (5) assuming perfect phase compensation.

\section{B. LLR Expression in an Urban Channel with Perfect CSI}

We then consider an urban propagation channel model and we firstly assume in this part that we have a perfect CSI. In this context, the symbol attenuation $a[k]$ and the noise power $\sigma^{2}{ }_{n_{I}}{ }^{\prime}$ are perfectly known at the receiver, assuming perfect channel estimation. These hypotheses are not always realistic for practical scenarios but allow us to derive the optimized detection function providing a lower bound on the best achievable decoding performance.

In addition, the phase is assumed perfectly compensated thus $\varepsilon_{\theta}=0$. From (5) the received symbol is thus modeled as:

$$
y[k]=a[k] x[k]+n_{I}{ }^{\prime}[k]
$$

From (11) we can write:

$$
p(y[k] / x[k])=\frac{1}{\sqrt{2 \pi \sigma^{2}{ }_{n_{I}}^{\prime}}} e^{\frac{-(y[k]-a[k] x[k])^{2}}{2 \sigma^{2}{ }_{n_{I}}}}
$$

And thus from (2) we can deduce that:

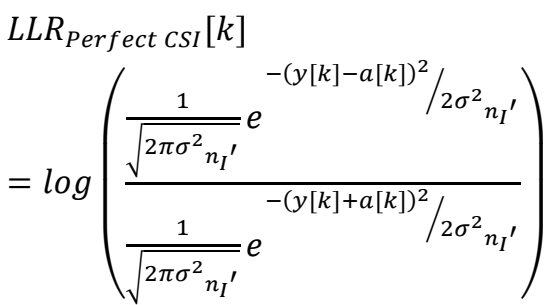


The final expression is given by:

$$
\operatorname{LLR}_{\text {Perfect } C S I}[k]=\frac{2 a[k] y[k]}{\sigma^{2}{ }_{n_{I}{ }^{\prime}}}
$$

Note that by taking the expectation of the scaling factor of the above expression with respect to the fading channel probability density function leads to the expression of the equivalent AWGN channel as given in (5) assuming only the knowledge of the average signal-to-noise ratio (without loss of generality, we assume here that $E[a[k]]=1$ ).

\section{LLR Expression in an Urban Channel with Partial CSI}

We then consider an urban propagation channel model assuming partial statistical CSI. The symbol attenuation $a[k]$ value is not known anymore but its statistical behavior is, meaning that the Probability Density Function (pdf) of $a$ is known as well as its related parameters (it implicitly assumes that the estimation of these parameters is possible in a practical scenario). In addition, the phase is assumed perfectly estimated thus $\varepsilon_{\theta}=0$.

The $L L R$ is derived from (2) according to the received symbol expression (5), and considering statistical partial CSI as follows:

$$
p(y[k] / x[k])=\int_{0}^{+\infty} p(y[k], a / x[k]) d a
$$

With :

$$
p(y[k], a / x[k])=\frac{1}{\sqrt{2 \pi \sigma^{2} n_{I^{\prime}}}} e^{\frac{-(y[k]-a x[k])^{2}}{2 \sigma^{2} n_{I^{\prime}}}} p(a)
$$

Thus :

$$
\begin{aligned}
& L_{L R} R_{\text {Statistical } C S I}[k] \\
& =\log \left(\frac{\int_{0}^{+\infty} p(y, a / x=1) d a}{\int_{0}^{+\infty} p(y, a / x=-1) d a}\right) \\
& =\log \left(\frac{\int_{0}^{+\infty} p(y / a, x=1) p(a) d a}{\int_{0}^{+\infty} p(y / a, x=-1) p(a) d a}\right)
\end{aligned}
$$

We finally have:

$$
\begin{aligned}
& L_{L} R_{\text {Statistical } C S I}[k] \\
& =\log \left(\frac{\int_{0}^{+\infty}\left(e^{\frac{-(y[k]-a)^{2}}{2 \sigma^{2} n_{I^{\prime}}}} p(a)\right) d a}{\int_{0}^{+\infty}\left(e^{\frac{-(y[k]+a)^{2}}{2 \sigma^{2} n_{I^{\prime}}}} p(a)\right) d a}\right)
\end{aligned}
$$

For the considered channel model, it can be assumed that the symbol duration being longer than the correlation duration of the direct signal component samples, the pdf of $a$ is equal to the pdf of $a$ knowing the direct signal component. In this way $a \sim \operatorname{Rice}\left(z, b_{0}\right)[4]$ and the complete expression of the $L L R$ is given by:

$$
\begin{aligned}
& L_{L} R_{\text {Statistical CSI }}[k] \\
& =\log \left(\frac{\int_{0}^{+\infty} e^{\frac{-(y[k]-a)^{2}}{2 \sigma^{2} n_{I}{ }^{\prime}}} \frac{a}{b_{0}} e^{-\frac{\left(a^{2}+z^{2}\right)}{2 b_{0}}} I_{0}\left[\frac{z a}{b_{0}}\right] d a}{\int_{0}^{+\infty} e^{\frac{-(y[k]+a)^{2}}{2 \sigma^{2} n_{I}{ }^{\prime}}} \frac{a}{b_{0}} e^{-\frac{\left(a^{2}+z^{2}\right)}{2 b_{0}}} I_{0}\left[\frac{z a}{b_{0}}\right] d a}\right)
\end{aligned}
$$

Where:

- $z$ is the direct signal component amplitude,

- $b_{0}$ is the average multipath power with respect to an unblocked direct signal,

- $\quad I_{0}\left[\frac{z a}{b_{0}}\right]=\int_{0}^{\pi} e^{\frac{z a}{b_{0}} \cos \theta} d \theta$ is the modified Bessel function of first kind and zero order.

The above expression has no closed form expression and it can be computationally prohibitive to evaluate for every soft inputs. If valuable, this approach is from far too complex for a practical application. Note also that in a real scenario, the different parameters have to be estimated.

\section{LLR Expression in an Urban Channel with No CSI}

We finally consider the case where no CSI is available at the receiver. The fading gain $a[k]$ is assumed unknown as well as its statistical behavior and the noise power is assumed unknown too.

The phase is firstly assumed to be perfectly estimated but it can be noticed that the method described below can be used with a phase estimation made by a Phase-Locked Loop (PLL) too. The results will be analysed in these two settings.

Motivated by the $L L R$ mathematical expression assuming an urban propagation channel and perfect CSI (14), and according to [2], we are looking for a linear expression of the $L L R$ expression considering an urban propagation channel without CSI. The idea is to find a method that can help to find the best linear approximation for an $L L R$ soft input avoiding 
the complexity burden induced by some statistical knowledge at the receiver end. The linear approximation can be written as a linear function of the observation sample $y[\mathrm{k}]$ as:

$$
L L R[k]=\alpha y[k]
$$

The method proposed in [2] consists in determining the scaling coefficient $\alpha$ that maximizes the mutual information between the transmitted symbol $x[k]$ and the detector input $L L R[k]$ which is a sufficient statistics:

$$
I(L L R ; X)=H(X)-H(X / L L R)
$$

Where $H(X)$ and $H(X / L L R)$ denote the entropy of a random variable $X$ and the conditional entropy of $X$ given $L L R$ respectively.

When considering binary input memoryless channels as in our case, this expression can be easily expressed as a function of the pdf of the LLR at the input of the receiver [2]:

$$
I(L L R ; X)=1-\int_{-\infty}^{+\infty} \log _{2}\left(1+e^{-L L R}\right) p(L L R) d L L R
$$

Using this expression a good choice for $\alpha$ is given by:

$$
\alpha_{M C L A}=\arg \max _{\alpha} I(L L R ; X)
$$

A simple line search algorithm can be used to evaluate the optimum value.

Originally in [2], the proposed optimization method to find the optimal $\alpha$ assumes the knowledge of the LLR's pdf. Unfortunately, this knowledge is not always available in practical situations. We aim to go a step further to find an efficient method for computing a good approximation of this quantity without any statistical knowledge.

If we first assume that the emitted symbol $x[k]$ is known, the mutual information between $X$ and $L L R$ can be approximated using a time average estimation by the following expression [3]:

$$
I(L L R ; X) \approx 1-\frac{1}{N} \sum_{k=1}^{N} \log _{2}\left(1+e^{-x[k] \alpha y[k]}\right)
$$

Where:

- $\quad N$ is the number of symbols used to estimate $I(L L R ; X)$.

Although computationally efficient, this method assumes the use of a learning sequence to evaluate the mutual information at the channel decoder input. Fortunately, this assumption can be relaxed. Indeed, if the emitted symbol $x[k]$ is unknown, the mutual information between $X$ and $L L R$ can be well approximated using the following expression [3]:

$$
\begin{aligned}
I(L L R ; X) \approx 1- & \frac{1}{N} \sum_{k=1}^{N}\left[-P_{e n}[k] \log _{2}\left(P_{e n}[k]\right)-(1\right. \\
& \left.\left.-P_{e n}[k]\right) \log _{2}\left(1-P_{e n}[k]\right)\right]
\end{aligned}
$$

Where:

$$
\text { - } \operatorname{Pen}[k]=\frac{e^{\frac{|\alpha y[k]|}{2}}}{e^{\frac{|\alpha y[k]|}{2}}+e^{-\frac{|\alpha y[k]|}{2}}}
$$

Thus, the obtained blind estimation is only dependent of the $L L R$ magnitude and independent of the emitted sequence.

Finally, once the coefficient $\alpha$ maximizing $I(L L R ; X)$ has been determined, the LLR can be deduced by:

$$
\operatorname{LLR}_{\text {No } C S I}[k]=\alpha y[k]
$$

This can be efficiently done by one dimensional line search algorithms.

We finally have an efficient method to compute a low complexity linear $L L R$ expression that enables to have near maximum mutual information between the emitted symbols and the linear approximated $L L R S$. The AWGN case appears as a special case when $\alpha$ is equal to the average signal to noise ratio. So we expect to have better performance using our method than using the default AWGN expression.

\section{Simulation RESUlts}

The detection function expressions adapted to an urban propagation channel have been derived for different levels of CSI in the last section and have been tested with the software SiGMeP described in [1] (see Fig. 1). The GPS L1C navigation message demodulation performance through the Prieto Land Mobile Satellite (LMS) propagation channel model, with these different detection function expressions, are provided and compared with the results obtained with the $L L R_{A W G N}$ used currently in GNSS receivers.
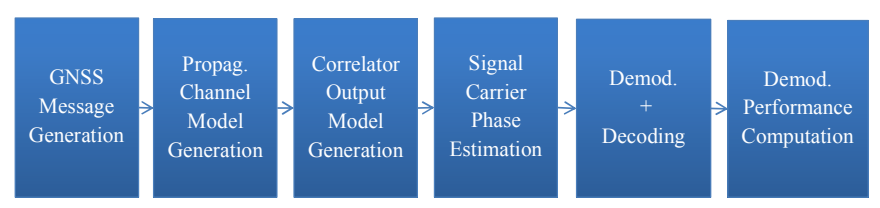

Fig. 1. Simulation tool SiGMeP structure definition.

\section{A. GPS L1C Navigation Message}

The GPS L1C navigation message (defined in [5]) consists of a continuously flow of frames and each frame is divided into 3 subframes. Subframe 1 is formed by 9 information bits and provides the Time Of Arrival (TOI). Subframe 2 is formed by 
600 information bits: 576 bits of non-variable data and 24 Cyclic Redundancy Check (CRC) bits. The data are nonvariant over a period of multiple frames and provide Clock correction and Ephemeris Data (CED). Subframe 3 is formed by 274 information bits: 250 bits of variable data and $24 \mathrm{CRC}$ bits. Fig. 2 illustrates the GPS L1C message structure.

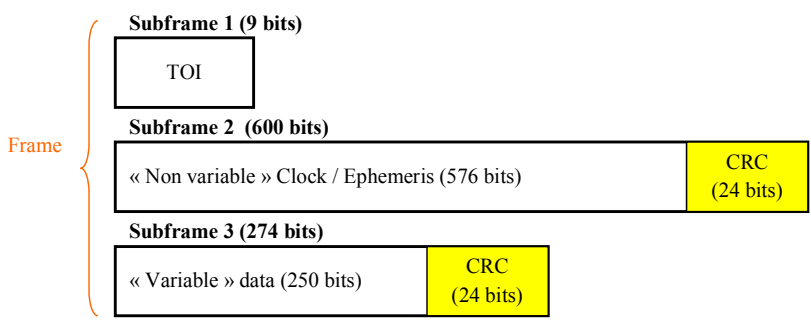

Fig. 2. GPS L1C data message description.

Subframe 1 is encoded by a BCH (Bose, Ray-Chaudhuri and Hocquenghem) code into 52 symbols.

Subframes 2 and 3 are encoded by a Low-density paritycheck (LDPC) code with code rate equal to $1 / 2$. The L1C standard [5] specifies a $(1200,600)$ systematic irregular LDPC code for subframe 2 and a $(548,274)$ systematic irregular LDPC code for subframe 3 . The LDPC codes for subframes 2 and 3 are different because of their different lengths. Finally, the 1748 encoded symbols are interleaved by a block interleaver of 38 arrows and 46 columns. The resulting frame consists of 1800 symbols transmitted at 100 sps. The entire GPS L1C navigation message lasts thus $18 \mathrm{~s}$.

The GPS L1C signal is divided into two components: the data and the pilot component. The power dedicated to the data component is $\frac{1}{4}$ of the transmitted signal total power and $\frac{3}{4}$ for the pilot component.

\section{B. Prieto Propagation Channel Model}

The Prieto propagation channel model designed in 2010 [4] is an evolution of the Perez Fontan model [6]. It is a LMS statistical narrowband model in urban environment adapted for the demodulation point of view. The Perez Fontan model was referenced in the European Cooperation in the field Of Scientific and Technical Research (COST) in 2002.

The model is narrowband, meaning that the delay of the direct signal and the delays of the multipath echoes are not modeled. The Channel Impulse Response (CIR) is thus modeled as:

$$
h(t ; \tau)=c(t) \delta\left(t-\tau_{\text {direct }}(t)\right)
$$

Where:

- $\quad c(t)=a_{\text {received }}(t) e^{j \varphi_{\text {received }}(t)}$ represents the channel attenuation and phase with $a_{\text {received }}(t)$ the received signal complex envelope.
The model is statistic and based on measurement campaigns carried out in the 90s. The received signal complex envelope distribution follows a Loo distribution.

The channel impact $c(t)$ of the overall received signal can be divided into two components, the direct signal and the multipath components:

$$
c(t)=a_{\text {direct }}(t) e^{j \varphi_{\text {direct }}(t)}+a_{\text {multipath }}(t) e^{j \varphi_{\text {multipath }}(t)}
$$

Where:

- $\quad a_{\text {direct }}(t)$ is the direct signal component amplitude and $\varphi_{\text {direct }}(t)$ is its Doppler phase,

- $\quad a_{\text {multipath }}(t)$ is the multipath component amplitude and $\varphi_{\text {multipath }}(t)$ is its phase.

The direct signal component corresponds to the Line-OfSight (LOS) signal which can be potentially shadowed or blocked. The multipath component corresponds to the sum of all the reflections/refractions of the transmitted signal found at the RF block output. [6]:

The distribution of the Loo parameters is defined as follows

- $\quad$ The amplitude of the direct signal component $a_{\text {direct }}(t)$ follows a Log-Normal distribution, characterized by its mean $M_{A}$ and its standard deviation $\Sigma_{A}$,

- $\quad$ The amplitude of the multipath component $a_{\text {multipath }}(t)$ follows a Rayleigh distribution, with a standard deviation $\sigma$. The value of $\sigma$ is calculated from the average multipath power with respect to an unblocked LOS signal: $M P(29)$. $M P$ is the parameter provided in the literature.

$$
\sigma=\sqrt{10 \frac{\mathrm{MP}}{10} / 2}
$$

The set of parameters $\left(M_{A}, \Sigma_{A}, M P\right)$ completely defines the Loo distribution and is referred as the Loo parameters. They follow Gaussian distributions which parameters depend on the environmental conditions:

- The type of environment (semi-urban, urban, deep urban...),

- The satellite elevation angle,

- The signal carrier band,

- The channel states.

The model classifies the received signal into two states, according to the impact level of the propagation channel. More specifically, each state corresponds to a particular environment configuration, representative to the strength of the shadowing/blockage effect on the received direct signal component:

- "Good" for LOS to moderate shadowing, and

- "Bad" for moderate to deep shadowing. 
The state transitions are dictated by a semi first-order Markov chain, the state durations following a Log-Normal distribution (see Fig. 3).

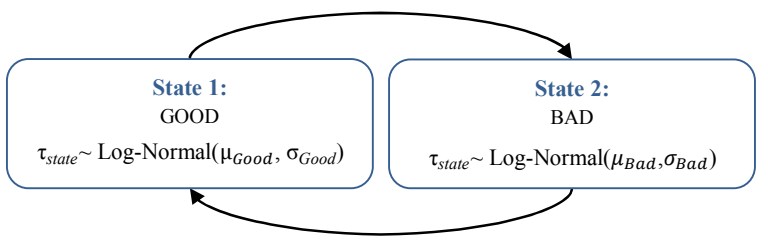

Fig. 3. Semi-Markov chain state transitions process.

The Prieto channel model parameters used in the SiGMeP simulator has been chosen for this article to represent a difficult signal reception condition configuration in an urban environment. They are listed in Table 1.

TABLE 1: LMS CHANNEL MODELS PARAMETERS USED FOR THE SIMULATIONS WITH SIGMEP

\begin{tabular}{|c|c|}
\cline { 2 - 2 } \multicolumn{1}{c|}{} & Prieto \\
\hline Environment type & Urban \\
\hline User Speed & $50 \mathrm{~km} / \mathrm{h}$ \\
\hline Band of the measurements & $\mathrm{S}-$ band \\
\hline Satellite Elevation & $40^{\circ}$ \\
\hline
\end{tabular}

At the time of the article's publication, the simulations were conducted assuming a S-band signal since the L-band Prieto channel model parameters [4] seemed to not represent faithfully the real propagation channel.

\section{Results}

The advanced detection function expressions adapted for an urban propagation channel and derived according to the CSI knowledge have been implemented in the simulator SiGMeP. The $L L R_{\text {statistical CSI function has not been integrated because }}$ of its complexity for a practical application. The Clock and Ephemeris Data (CED) Error Rate (CEDER), contained by subframe 2 for GPS L1C (being the only necessary data to compute a first position) has been computed with each of the detection function expressions and compared.

The results obtained with the $L L R_{A W G N}$ function represent the performance provided by a current GNSS receiver for GPS L1C with an urban channel model, with perfect phase estimation.

The results obtained with the $L L R_{\text {perfectCSI }}$ function are not really representative of the reality because it is not possible to perfectly estimate the fading gains of the urban channel. This case has to be seen as a lower bound on the achievable performance.

Then, the demodulation performance has been computed with both detection function expressions: $L L R_{\text {no CSI }}$ known corresponding to no CSI but assuming that the emitted symbol $x[k]$ is known (24), and LLR no CSI $x$ unknown corresponding to no CSI and assuming that the emitted symbol $x[k]$ is unknown (25), thanks to the proposed method (see section III.D). The coefficient $\alpha$ has been computed for each LDPC codeword: subframe 2 and subframe 3 separately.

Fig. 4 represents thus GPS L1C demodulation performance (CEDER as a function of $\mathrm{C} / \mathrm{N}_{0}$ ), obtained with the simulator SiGMeP with the Prieto channel model and perfect phase estimation, using the detection function expressions described before and adapted to an urban channel.

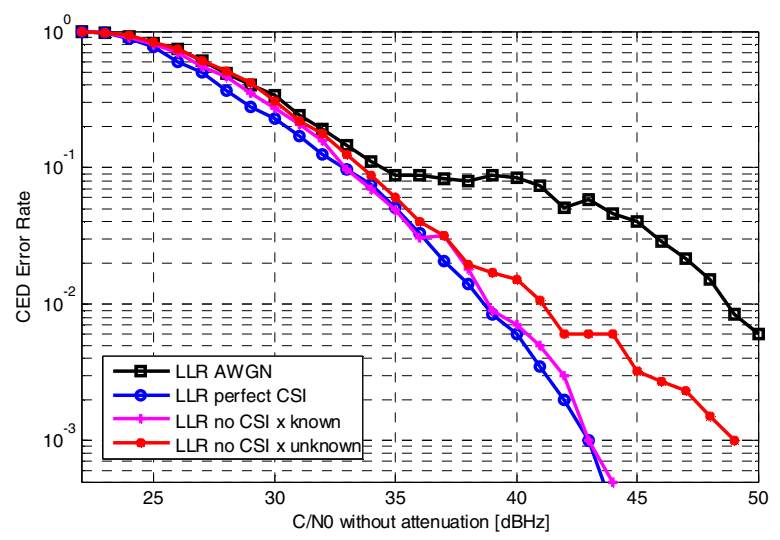

Fig. 4. GPS L1C demodulation performance in the Prieto channel model with perfect phase estimation for advanced detection functions adapted to an urban channel.

It can be noticed that to achieve an error rate of $10^{-2}$ with GPS L1C in an urban propagation channel with perfect phase estimation, a classical receiver (with the $L L R_{A W G N}$ function) needs a minimal $\mathrm{C} / \mathrm{N}_{0}$ value equal to $48.7 \mathrm{~dB}-\mathrm{Hz}$, whereas if the advanced detection function $L L R_{\text {no CSI } x \text { unknown }}$ is used, this minimal $\mathrm{C} / \mathrm{N}_{0}$ value decreases until $39.5 \mathrm{~dB}-\mathrm{Hz}$. It means that if $L L R_{\text {no CSI x unknown }}$ is used instead of $L L R_{A W G N}$ the demodulation performance is improved by approximately $9 \mathrm{~dB}$ in this case, needing absolutely no additive knowledge of CSI compared to the $L L R_{A W G N}$ case.

Fig. 5 presents the same results, with the difference that the phase estimation is made by a PLL, inducing a phase estimation error $\varepsilon_{\theta} \neq 0$.

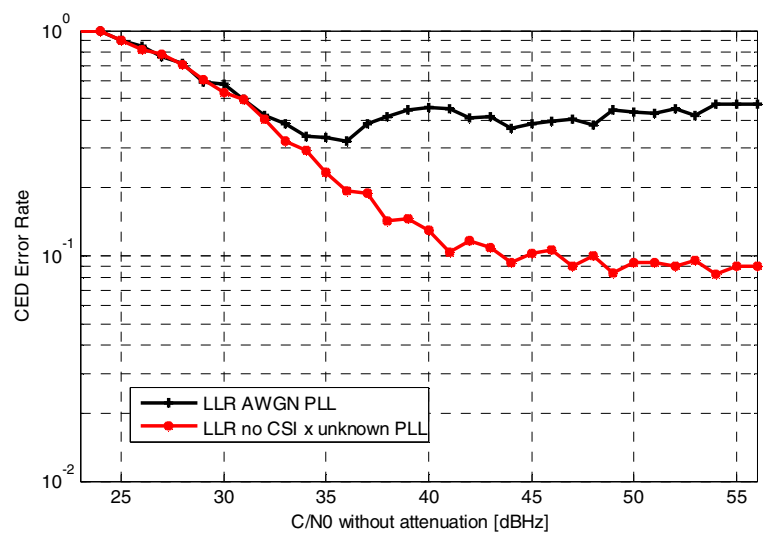

Fig. 5 : GPS L1C demodulation performance in the Prieto channel model with PLL phase estimation for advanced detection functions adapted to an urban channel.

If a PLL phase tracking is considered as it is shown in Fig. 5 , the use of the advanced detection function $L L R_{\text {no CSI } x \text { unknown }}$ adapted to an urban propagation channel 
and assuming no CSI instead of the detection function $L L R_{A W G N}$ assuming an AWGN propagation channel, brings GPS L1C demodulation performance improvement. However, the floor existing in the $L L R_{A W G N}$ case is always present in the $L L R_{\text {no } C S I} x$ unknown case.

\section{CONCLUSION}

This article proposes a computationally efficient advanced processing algorithm that significantly improves the receiver sensibility in urban propagation channels. We have demonstrated that the use of a refined detection function adapted to the urban environment considerably increases the demodulation performance of GNSS signals. For a CEDER of $10^{-2}$ a decoding gain of approximately $9 \mathrm{~dB}$ is obtained between the detection function corresponding to the AWGN propagation channel and the advanced detection function corresponding to an urban propagation channel and considering no CSI at all, with ideal phase estimation.

The simulations have been done with LDPC channel codes (computation of GPS L1C demodulation performance), but it is important to notice that the advanced detection functions derived in this article can be applied to all soft input decoders such as Viterbi decoder for trellis based codes, the MAP/BCJR decoding for turbo-codes and the Belief Propagation decoding for LDPC codes.
Nevertheless, the demodulation performance obtained with a PLL phase tracking in urban environments (which is the realistic case) is not satisfactory, even if an improvement has been brought by the advanced detection function. Future works have to be conducted to further assess the impact of PLL phase error on GNSS signals demodulation performance in urban areas.

\section{REFERENCES}

[1] M. Roudier, A. Garcia-Pena, O. Julien, T. Grelier, L. Ries, C. Poulliat, M.-L. Boucheret, and D. Kubrak, "New GNSS Signals Demodulation Performance in Urban Environments," in Proceedings of the 2014 International Technical Meeting of The Institute of Navigation, 2014.

[2] W. Ryan and S. Lin, Channel Codes: Classical and Modern. Cambridge University Press, 2009.

[3] S. ten Brink, Design of concatenated coding schemes based on iterative decoding convergence. Aachen: Shaker, 2002.

[4] R. Prieto-Cerdeira, F. Perez-Fontan, P. Burzigotti, A. Bolea-Alamañac, and I. Sanchez-Lago, "Versatile two-state land mobile satellite channel model with first application to DVB-SH analysis," Int. J. Satell. Commun. Netw., vol. 28, no. 5-6, pp. 291-315, 2010.

[5] US Government, "INTERFACE SPECIFICATION IS-GPS-800 Navstar GPS Space Segment/User Segment L1C Interface.” Sep-2012.

[6] F. Perez-Fontan, M. A. Vázquez-Castro, S. Buonomo, J. P. PoiaresBaptista, and B. Arbesser-Rastburg, "S-band LMS propagation channel behaviour for different environments, degrees of shadowing and elevation angles," IEEE Trans. Broadcast., vol. 44, no. 1, pp. 40-76, 1998.

[7] F. P. Fontan, M. Vazquez-Castro, C. E. Cabado, J. P. Garcia, and E. Kubista, "Statistical modeling of the LMS channel," IEEE Trans. Veh. Technol., vol. 50, no. 6, pp. 1549-1567, 2001. 